\title{
Políticas públicas del Gobierno de Ecuador que benefician a la primera infancia
}

\section{Public policies of the Government of Ecuador that benefit the first childhood}

Vinicio Alexander Chávez Vaca

Universidad Internacional del Ecuador, Ecuador

Susana Iselda Delgado Santos

Consultor Independiente, Ecuador

Autor para correspondencia: vchavez@uide.edu.ec ; susys.delgado@hotmail.com

Fecha de recepción: 21 de Noviembre de 2017 - Fecha de aceptación: 15 de Abril de 2018

Resumen: En la presente investigación se propuso describir cómo las políticas públicas implementadas en Ecuador han beneficiado a los niños de 0 a 5 años, entendida como la primera infancia en el país andino. Desde una perspectiva cuantitativa, para el logro de este objetivo se utilizó como uno de los principales métodos el Bibliográfico Documental, y las técnicas de la revisión bibliográfica documental y la Encuesta; la primera posibilitó la identificación de los principales conceptos de políticas públicas y desarrollo infantil integral, así como la acepción e interpretación práctica que ha generado en Ecuador este tema de la agenda internacional. El estudio empírico permitió conocer la apreciación al respecto de los especialistas de los Centros Infantiles del Buen Vivir, creados por el Estado para fortalecer el cuidado de los niños preservando sus derechos. Luego de la discusión establecida entre la fundamentación teórica y la práctica, se concluye que, si bien se dispone de políticas públicas que han favorecido el desarrollo integral de los niños, con una marcada incidencia en la familia y sobre su conocimiento en temas como equidad, inclusión e igualdad de oportunidades, aún existen limitaciones sobre todo de los medios didácticos que beneficien las actividades desarrolladas en esas instalaciones. De ahí que se recomiende un estudio metodológico más riguroso sobre el asunto y se haga un llamado a las instituciones gubernamentales para no escatimar en recursos materiales y humanos que sigan promoviendo la calidad de vida de la primera infancia.

Palabras Claves: desarrollo infantil; primera infancia; política pública; educación infantil

Abstract: The present investigation aimed to describe how public policies implemented in Ecuador have benefited children from 0 to 5 years old, understood as early childhood in the Andean country. From a quantitative perspective, for the achievement of this objective, one of the main methods was the Documentary Bibliography, and the techniques of the documentary bibliographic review and the Survey; the first allowed the identification of the main concepts of public policies and integral child development, as well as the acceptance and practical interpretation that this topic of the international agenda has generated in Ecuador. The empirical study allowed to know the appreciation of the specialists of the Good Living Children's Centers, created by the State to strengthen the care of children by preserving their rights. After the discussion established between the theoretical foundation and practice, it is concluded that, although there are public policies that 
have favored the integral development of children, with a marked impact on the family and on their knowledge on issues such as equity, inclusion and equality of opportunities, there are still limitations especially of the didactic means that benefit the activities developed in those facilities. Hence, a more rigorous methodological study on the subject is recommended and a call is made to government institutions to spare no material and human resources that continue to promote the quality of life of early childhood.

Key Words: child development; early childhood; public policy; children's education

\section{Introducción}

En fecha tan tardía como el año 1990, con la firma de la Convención sobre los Derechos del Niño, fue que el mundo reconoció por primera vez la importancia del bienestar de los infantes. Este instrumento internacional fue resultado de un largo proceso protagonizado por organismos que unieron esfuerzos en la lucha por preservar y mejorar la calidad de vida en la niñez, luego de siglos en el que poco se trataron esos temas en el contexto mundial.

Con posterioridad y hasta la fecha, el desarrollo del niño ha sido motivo de preocupación y debate no solo en el escenario internacional sino al interior de los países, que además de adoptar leyes en referencia a los derechos de la infancia, formulan e implementan políticas públicas que intentan garantizar esos principios en la práctica. En la actualidad incluso se habla en términos de desarrollo integral de la infancia, lo que supone un movimiento mayor de recursos humanos, organismos y recursos en función de que el niño crezca de forma confortable.

Para ello ha de partirse de un proceso de diagnóstico de las formas de vida de los infantes, de modo que en la intervención se priorice a los pequeños que se encuentran siendo discriminados, marginados y desfavorecidos. Sin embargo, por lo general, aunque las políticas son pensadas para todo tipo de realidades, en la práctica muchas veces no llega a todos los beneficiarios ni a todos los lugares que requieren de las estrategias de mejoramiento humano.

Esto tiene que ver con que los objetivos planteados no resultan correctamente planificados dentro de las líneas de acción de la estrategia, porque no cuenta con el respaldo financiero necesario para el empeño previsto, o porque los decisores u organismos que participan no alcanzan la cohesión necesaria para implementar las actividades en beneficio de los infantes; otras veces no funciona el sistema de promoción y divulgación de la información en el Gobierno y la población, ni se implementan mecanismos de control que permitan evaluar la efectividad de las actividades y actualizar en caso necesario.

De acuerdo con Dodge (2015), contrario a lo que por décadas se defendió, el desarrollo del niño no depende solo de la capacidad psicopedagógica, sociocultural y económica de los padres, sino de un entramado de factores mucho más complejo que de no entrelazarse entre sí pueden dejar desprotegidos a los infantes en alguna de las dimensiones de la vida, de ahí que el autor explique que "la buena salud (de la madre y el niño), la buena alimentación, buenas prácticas de crianza, apoyos sociales sólidos e interacción estimulante con personas ajenas al hogar se conciertan para facilitar la mejor oportunidad de éxito" (p. 7). 
Coincidiendo con esta idea, otros autores entienden el desarrollo infantil integral como un fenómeno influido por la calidad de la salud, la educación, el entorno donde crece el niño; es decir, depende de la participación de diferentes sectores de la economía de un país (Moreno, 2016), y de satisfacer otras necesidades importantes como el sentimiento de protección que demanda el niño, la afectividad, la nutrición, la recreación, la identidad; lo cual supone contar con una infraestructura millonaria.

En las últimas décadas Ecuador ha impulsado una labor en función de los derechos del niño, logrando destacarse entre los países de la región. Lo primero que hizo el Estado fue otorgarle a la familia el papel determinante del desarrollo humano integral, como el eje transversal de las políticas para el ejercicio de los derechos de los niños; sin embargo, a la responsabilidad del Gobierno fue mayor a partir de 2012 cuando el MIES (2013) declara que el Desarrollo Infantil Integral como una política prioritaria de Estado, universal y obligatoria.

El caso ecuatoriano resulta más halagüeño en tanto la agenda nacional en relación a la infancia, desde inicio de este milenio, ha insistido en ofrecerle un enfoque de equidad de género e inclusión a sus planes de acción, lo que supone incluir en todas sus ofertas a niñas y niños sin importar las condiciones culturales y sociales de las familias, pueblos y comunidades. El Estado intenta intervenir en los diferentes contextos para ofrecer iguales derechos y garantías a los pequeños.

\section{Infancia}

El concepto de infancia ha tenido varias definiciones, pero es en la época moderna donde se ha ofrecido una conceptualización más primordial a esta etapa de la vida humana. Según Berlinski y Schady (2015), fenómenos como la disminución de la mortalidad infantil, el desarrollo y ampliación del sistema educativo, y el papel ofrecido a la familia por la sociedad, influyeron en la nueva perspectiva dada a la primera infancia. Sin embargo, cuando en el siglo XVIII se intensificó el proceso de industrialización, entre los más explotados se encontraban los niños.

En el siglo XIX no eran pocos los organismos que veían al infante como objeto de compasión o de la filantropía, se reconocía que la infancia era un período de la vida que requería protección y que era adecuado que los Gobiernos intervinieran a favor de los niños (Lowe, 2004). No obstante, aunque en ese entonces el Estado comienza a entender su responsabilidad de proteger el bienestar de los niños, aún era la familia la que asumía la mayoría de las acciones para el desarrollo de sus hijos, por lo que no era de extrañar que la inmensa cantidad de los infantes heredara las mismas condiciones de pobreza y enfermedad de los padres.

De acuerdo con los analistas, la siguiente centuria devendría "el siglo de los niños", pues en 1924 la Organización de las Naciones Unidas daría a conocer el primer documento que reconocía los derechos de la infancia: la Declaración de Ginebra sobre los Derechos del Niño. Dos décadas después se crearía el Fondo de las Naciones Unidas para la Infancia (UNICEF). El tratamiento a los temas de la niñez se convertiría en uno de los principales asuntos de la agenda internacional. 
El gran suceso fue cuando en 1989, 140 países firmaron el documento de la Convención sobre los Derechos del Niño.

Aunque la mayoría de los autores se refieren a la primera infancia como la etapa comprendida entre los 0 y 8 años, el concepto puede cambiar de acuerdo a lo que determinen los países, como Ecuador, que presupone ese periodo como "el desarrollo integral de las niñas y los niños menores de 5 años" (Espinel, 2016, p. 1). Sin embargo, la Convención sobre los Derechos Humanos, al acoger el primer rango de edades, obliga a las naciones partes a asumir las responsabilidades de los pequeños de acuerdo con la primera definición (Comité de los Derechos del Niño, 2006).

Los estudios confirman la importancia que tienen los primeros años de vida para el desarrollo integral del niño, pues es en ese periodo donde el pequeño alcanza las primeras habilidades que incidirán en el resto de su vida como es pensar, sentir, moverse y aprender (UNICEF, 2010). Por tanto, a consideración de Moreno (2016), en los primeros años de la existencia el desarrollo cognitivo, lingüístico, social, emocional y motor resulta mucho más acelerado que en los años siguientes, por lo que la preocupación por un proceso natural del desarrollo integral de los infantes debe darse con una mayor garantía en esta etapa.

\section{Políticas públicas en relación a la infancia}

Las políticas públicas han sido definidas como el "conjunto de decisiones interrelacionadas, tomadas por un actor o grupo de actores respecto de la selección de metas y medios para alcanzarlas en una situación específica, y donde aquellas decisiones están dentro del ámbito de autoridad de esos actores" (Olavarría, 2007, p. 7). Para Moreno (2016) las políticas públicas implican aquellas decisiones que toma el Estado en función de alcanzar metas que por lo general suponen un propósito de mejoramiento social de los ciudadanos.

Mucho se ha avanzado a nivel internacional en la puesta en práctica de políticas públicas cuyo objetivo mayor ha sido la protección y beneficio de los infantes, y en esa labor, no solo se han logrado involucrar a los organismos e instituciones estatales, sino que también las estructuras privadas ejercen una importante influencia en elevar los estándares de desarrollo de la primera infancia, aunque sigue siendo el Gobierno el mayor responsable en este sentido. Y es que las condiciones de vida de los niños muchas veces guardan relación con el estado de pobreza y desigualdad en que se encuentra la vida de los infantes, por lo que la formulación de políticas públicas no solo incide en disolver los problemas sociales en el que se involucran los niños sino también sus familiares (MIES, 2013), por lo que la capacitación e participación de progenitores, tutores y cuidadores de los niños debe constituir el eje central de esta política pública (UNESCO, 2012).

Otras de las determinantes del éxito de las políticas públicas radican en el desarrollo de los sistemas de la salud y la educación en los países; y aunque los países han entendido la importancia de invertir en los programas sociales que atañen a estos sectores, aún existen países cuyos sistemas no logran satisfacer la demanda de las poblaciones más pobres y marginadas. En el caso de la atención sanitaria, persisten desafíos en la esperanza de vida al nacer y la prevención de enfermedades. En el aspecto educativo, la accesibilidad y obligatoriedad escolar 
constituyen asignaturas pendientes. En otros países, estos sectores se encuentran mayormente privatizados por lo que la posibilidad de un cambio resulta más lejana.

Para Berlinski y Schady (2015) "las políticas públicas deberían mejorar las vidas de los niños desde el momento de su concepción hasta mucho después de que hayan comenzado la escuela", y para ello, según Carneiro y Heckman (2003) existe un camino en que solo logrando una perspectiva integral se puede incidir en un cambio verdadero de las condiciones de vida de los niños que garanticen un desarrollo efectivo:

La información y el coaching (realizar campañas de concientización, instruir a las madres sobre la lactancia materna), la legislación (la licencia por maternidad/paternidad, la educación obligatoria a una determinada edad), las regulaciones (normas relativas a la publicidad de las leches en polvo para bebés, o sobre la proporción niños-personal en los jardines de cuidado infantil), las transferencias (las asignaciones universales por hijo, los créditos fiscales, las transferencias condicionadas) y los precios (jardines de cuidado infantil subsidiados, vacunas gratuitas) (p. 19).

Berlinski y Schady (2015) concluyen que el bienestar de los niños no se logra instantáneamente sino que constituye un proceso acumulativo del progreso infantil que incluye cuatro dimensiones: desarrollo físico, lenguaje/comunicación, destrezas cognitivas y habilidades socioemocionales: "Este proceso no se genera por sí solo sino que es moldeado por las experiencias que los niños acumulan en el hogar, en los jardines de cuidado infantil y en la escuela" (p. 35), al menos cuando de primera infancia se habla.

En esa tarea de asegurar el desarrollo integral de la primera infancia participan las familias, los docentes y las instituciones gubernamentales, entendiendo que el bienestar de la infancia garantiza el modo en que el futuro de las sociedades se vierte sobre el Estado, además de que el desarrollo de la infancia no solo constituye una meta de garantía económica, sino que supone un empeño ético de acuerdo con los principios humanos que el hombre ha logrado defender en la arena internacional.

\section{El caso ecuatoriano}

El esfuerzo por defender los derechos del niño en Ecuador y trabajar por su desarrollo integral iniciaría su momento cumbre en la década de 1980, pero las acciones en relación a esta meta internacional vendrían a consolidarse diez años después con la firma de la Convención de los Derechos del Niño en 1990. A partir de entonces surgirían numerosas organizaciones que dedicarían esfuerzos en ver a los niños como "sujetos de derechos, transformando viejas relaciones autoritarias que excluían de la toma de decisiones a los niños y a las niñas bajo el supuesto de que eran "menores" de edad" (ODNA, 2012, p. 41), y en esta misma perspectiva tratarían de involucrar a la sociedad.

Hasta la fecha, el cambio de perspectiva sobre la niñez en Ecuador ha supuesto el enfrentamiento a los altos índices de pobreza, desnutrición y mortalidad infantil, el analfabetismo, los actos de discriminación y marginalidad, la violencia familiar, y otros problemas sociales, que tuvo en la creación del Foro de la Niñez y Adolescencia en el país un 
momento importante, aunque con anterioridad la reforma del Código de Menores, en 1992, significaría el primer paso para accionar en pos del desarrollo de la primera infancia, en tanto incorporó en la nación la idea de los niños y las niñas y a los adolescentes como sujetos de derecho. Moreno (2016) asegura que partir de este momento las políticas públicas en relación a la infancia comienza a ser más notorias en el país.

Cáceres (2011) menciona momentos importantes sobre el accionar del Gobierno para la protección de la niñez, entre ellos, la fundación del Sistema Único de Atención a la Infancia (SUAI), alrededor del cual se articularon múltiples sectores en la atención de desarrollo infantil en la primera infancia con el fin de unificar metodologías y ampliar el alcance de estos servicios; y la creación del programa Creciendo con Nuestros Hijos (CNH) y los Centros Infantiles del Buen Vivir (CIBV), que incidirían en la capacitación de la familia en función del cuidado de los niños y para el cual se involucró un número no pequeño de profesionales de la educación y la salud.

De acuerdo con el propio autor, un paso agigantado se dio en 2003 con la publicación del Código de la Niñez y Adolescencia, que "estableció la obligatoriedad de definir y aprobar una Política Nacional de Protección Integral y un nuevo marco jurídico institucional de carácter descentralizado y participativo" (p. 11). A partir de ese momento los planes de acción del Gobierno con el objetivo del desarrollo integral del niño y la niña resultó más evidente y sistemático, y tuvo una apoyatura legislativa con la promulgación del Sistema de Protección Integral de la Infancia y del Consejo Nacional de Niños, Niñas y Adolescentes (CNNA), que según la ODNA (2012) partiría de tres principios de protección de derechos: "la paridad para la construcción de políticas públicas; la creación de instancias de protección; y la oferta de servicios que ejecuten las medidas de protección" (p. 42).

Más recientemente la Secretaría Nacional de Planificación y Desarrollo (SENPLADES, 2015) ha asegurado que el país se moviliza para la consolidación de la política intersectorial de primera infancia "como eje fundamental en la reducción de la inequidad socio-económica del país" (p.1). La no exclusión y el desarrollo integral de niños y niñas se encuentra entre los objetivos que planteaba el Plan Nacional del Buen Vivir 2013-2017, y que ha tenido una visualización práctica con la Estrategia Nacional Intersectorial para la Primera Infancia.

En 2016 el Ministerio de Coordinador de Desarrollo Social señalaba el incremento de la inversión para el trabajo intersectorial entre los ministerios de Inclusión Económica y Social, Educación y Salud, los cuales han provisto de los servicios elementales a millones de infantes menores de cinco años. En este sentido, el organismo ofrece igual relevancia a la familia que a la preparación docente pues de ahí dependen las estrategias en beneficio y desarrollo integral de los pequeños. Sin embargo, ¿Hasta qué punto se ha logrado la preparación de estas dos instituciones sociales para garantizar la protección de los derechos del niño? Hacia ese propósito va encaminado el presente estudio, consistente en describir la incidencia de los recursos materiales y humanos en beneficio de la primera infancia en Ecuador.

\section{Material y métodos}


Describir la incidencia de los recursos materiales y humanos en beneficio de la primera infancia suponen un enfoque cuantitativo de una investigación de tipo descriptiva, en tanto se pretende demostrar el alcance que ha manifestado la implementación de las políticas públicas en la preparación de padres y docentes que atienden la niñez en el periodo de 0 a cinco años de vida.

- Método: Como método fundamental de investigación se empleó el Bibliográfico Documental, que, a partir de la técnica de la revisión bibliográfica documental, favorece a la indagación acerca de conceptos como políticas públicas e infancia desde los enfoques más actuales como el de los derechos humanos. La exploración literaria ha permitido describir la evolución legal y práctica de las acciones que en Ecuador se implementan en pos del desarrollo integral del niño, ha permito conocer además cuáles han sido los resultados en el cuidado de los derechos de la infancia en el país, sus logros y limitaciones en el orden material y humano.

- Técnica: Para captar, de forma rápida, la visión que sobre los programas relacionados con el cuidado y protección de los derechos de niñas y niños menores de cinco años, tienen los docentes, como uno de los actores sociales más importantes involucrados en esa gestión, en la presente investigación se procedió al uso de la técnica de la Encuesta, con la ayuda del instrumento del cuestionario, compuesto por preguntas cerradas, con el objetivo de describir la incidencia de las políticas públicas en beneficio de la primera infancia.

- Población y muestra: En aras de ganar en tiempo y de que la investigación resultara viable, el estudio se concentró en Quito, que cuenta con aproximadamente 250 Centros Infantiles del Buen Vivir (CIBV). El objeto del presente estudio lo conforman los especialistas que laboran en cinco centros de esa localidad: el CIBV "San Francisco de Quito", el CIBV "Llano Grande”, el CIBV “José María García”, el CIBV "Nuevo Amanecer" y el CIBV "Isabel Vendramini”.

Como la población determinada para el presente estudio no es tan numerosa, y todos los especialistas tienen las mismas condiciones para resultar seleccionados, el cuestionario se aplicó a 45 profesionales, entre los que predominan las educadoras, seleccionados por un muestreo aleatorio simple. Sus respuestas trataron acerca de la calidad del servicio de esas instituciones que trabajan por el desarrollo infantil integral; la igualdad de oportunidades para niños y niñas, así como la perspectiva de inclusión de esas instituciones y fortalecimiento del respeto de los derechos de la niñez, lo cual permitirá apreciar si la inversión y los mecanismos estatales se corresponden con las garantías que en Ecuador quiere dársele al desarrollo integral infantil.

- Validez del instrumento aplicado: El instrumento se encuentra conformado por diez preguntas (Ver Anexo 1), cuya validación quedó en manos del juicio de expertos, dos docentes que alcanzan el grado de máster en Ciencias de la Educación y uno que posee el grado de Doctor en Ciencias de la Educación. La mayoría de las preguntas fueron catalogadas como buenas y excelentes, y plantearon sugerencias para la reformulación del cuestionario. Teniendo en cuenta la opinión de los expertos se procedió a la realización de los ajustes requeridos en el cuestionario, para ser aplicado a la muestra de esta investigación. 
La triangulación de datos se utilizó como método de contrastación de resultados con el objetivo de describir la incidencia de los recursos materiales y humanos provistos a través de políticas públicas del Estado ecuatoriano en beneficio de la primera infancia.

\section{Resultados}

Con el objetivo de describir la incidencia de los recursos materiales y humanos que a partir de políticas públicas el país ha puesto a disposición del beneficio de la primera infancia, se procederá a exponer los resultados devenidos de la aplicación de la encuesta a un total de 45 profesionales que trabajan en los Centros Infantiles del Buen Vivir "San Francisco de Quito", "Llano Grande", "Palestina", "Nuevo Amanecer" y "Mis Pequeños Angelitos", en Quito. Una vez seleccionada la muestra, el sistema de SPSS facilitó el procesamiento de los datos.

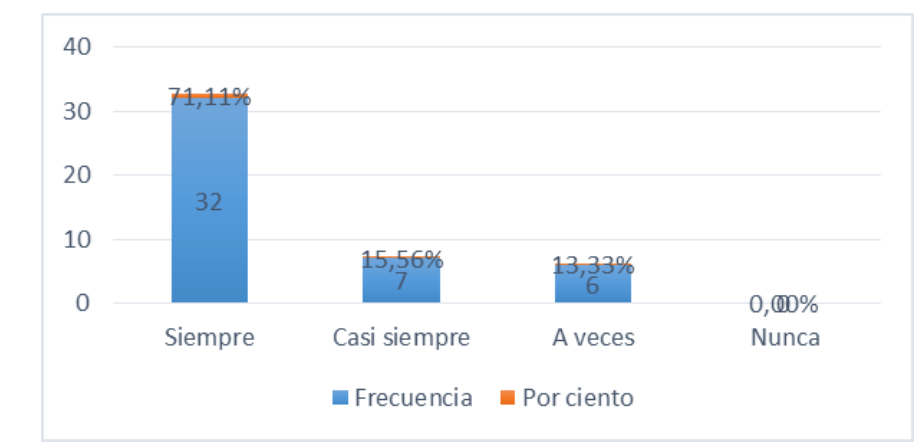

Figura 1: Atención a la niñez es igual a desarrollo integral infantil

De acuerdo con las respuestas de los profesionales que trabajan en los CIBV, la mayoría de esos especialistas creen que cuando los niños y niñas que reciben una adecuada atención durante su primera infancia, aumentan sus oportunidades de desarrollo integral siempre, 32 para un $71,11 \%$, o casi siempre, 7 para un $15,56 \%$. Solo seis educadoras, $13,33 \%$ plantearon que sucede a veces, ninguna afirmó que nunca sucede.

Tabla 1. Atención a la niñez es igual a desarrollo integral infantil

\begin{tabular}{lrr}
\hline Opciones & Frecuencia & Por ciento \\
\hline Siempre & 32 & $71,11 \%$ \\
Casi siempre & 7 & $15,56 \%$ \\
A veces & 6 & $13,33 \%$ \\
Nunca & 0 & $0,00 \%$ \\
\hline \multicolumn{2}{c}{ Fuente: Elaboración propia }
\end{tabular}

Estas afirmaciones tienen que ver con las experiencias positivas que ha traído la asistencia a los padres y tutores sobre los cuidados de los niños menores de cinco años en esas instituciones creadas por el Gobierno ecuatoriano para perfeccionar la atención y desarrollo infantil integral con el objetivo de beneficiar a la primera infancia y el futuro de los infantes. Esos centros tienen orientado la capacitación de los progenitores y la influencia en los infantes sobre temas como los derechos humanos de los niños, la alimentación, salud y nutrición de los infantes, la educación y afectividad, es decir, abarca todas las dimensiones de la vida infantil como el principio de un crecimiento sano y confortable. 


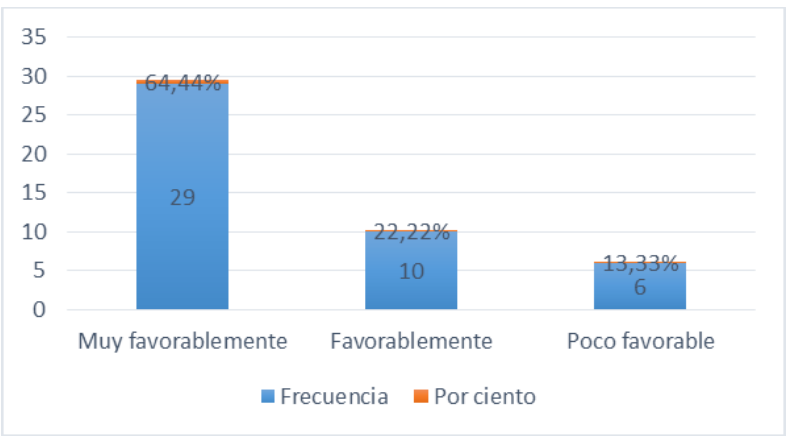

Figura 2. Incidencia en los padres

La mayoría de los encuestados entiende que el programa de los CIBV ha incidido muy favorablemente en la preparación de los padres y cuidadores de los niños en la calidad de vida y desarrollo integral de los infantes (29 para un 64,44\%); 10 personas $(22,22 \%$ ) opinaron que ha sido favorable el resultado de orientar e influir en los padres para la crianza de los hijos, y solo seis opinaron que los saldos han sido poco favorables $(13,33 \%)$.

Tabla 2. Incidencia en los padres

\begin{tabular}{lrr}
\hline Opciones & Frecuencia & Por ciento \\
\hline Muy favorablemente & 29 & $64,44 \%$ \\
Favorablemente & 10 & $22,22 \%$ \\
Poco favorable & 6 & $13,33 \%$ \\
\hline
\end{tabular}

Fuente: Elaboración propia

La importancia de las políticas públicas dirigidas a la atención y desarrollo integral de la niñez en Ecuador radica en que los padres, incluso los que se encuentran en condiciones de pobreza, han entendido acerca de temas como la diversidad cultural, la equidad de género e inclusión, así como las necesidades educativas especiales, para ejercer una responsable crianza de los hijos. De ahí que sea muy favorable el resultado de centros como los CIBV en la mejora de la calidad de vida de los infantes.

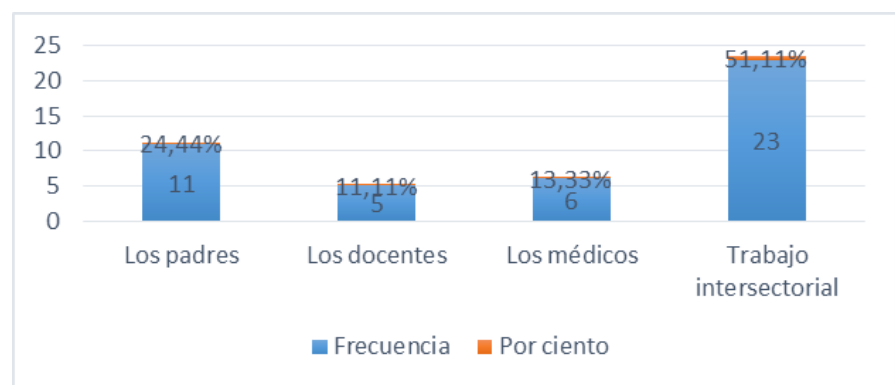

Figura 3. El desarrollo integral de los niños depende de...

Otros de los saldos positivos de la Encuesta ha sido que más de la mitad de los especialistas que trabajan en los CIBV han entendido que el desarrollo integral de los niños depende de un trabajo intersectorial (23 para un 51,1\%). No obstante, aun 11 personas $(24,44 \%)$ opina que el desarrollo de los hijos depende solo de los padres, mientras que cinco $(11,11 \%)$ creen que depende de los maestros y seis $(13,33 \%)$ entienden que son los médicos los más responsables de la vida de los infantes. 
Tabla 3. El desarrollo integral de los niños depende de...

\begin{tabular}{lrr}
\hline Opciones & Frecuencia & Por ciento \\
\hline Los padres & 11 & $24,44 \%$ \\
Los docentes & 5 & $11,11 \%$ \\
Los médicos & 6 & $13,33 \%$ \\
Trabajo intersectorial & 23 & $51,11 \%$ \\
\hline \multicolumn{2}{c}{ Fuente: Elaboración propia }
\end{tabular}

La incidencia de las políticas públicas implementadas por el Gobierno ecuatoriano en relación al desarrollo infantil integral ha extendido la comprensión de que los niños no son solo responsabilidad de los padres, como anteriormente al sigo XX se pensaba. Aunque ciertamente en la primera infancia las instituciones que más participan en la atención de los infantes son la familia, la escuela y el centro médico, hay que señalar que la vida de los niños depende incluso de los sectores de producción, desde lo que tienen que ver con la alimentación o los que garantizan el empleo de los progenitores y cuidadores, es decir, que el futuro de los pequeños es resultado de un trabajo multisectorial de toda la sociedad.

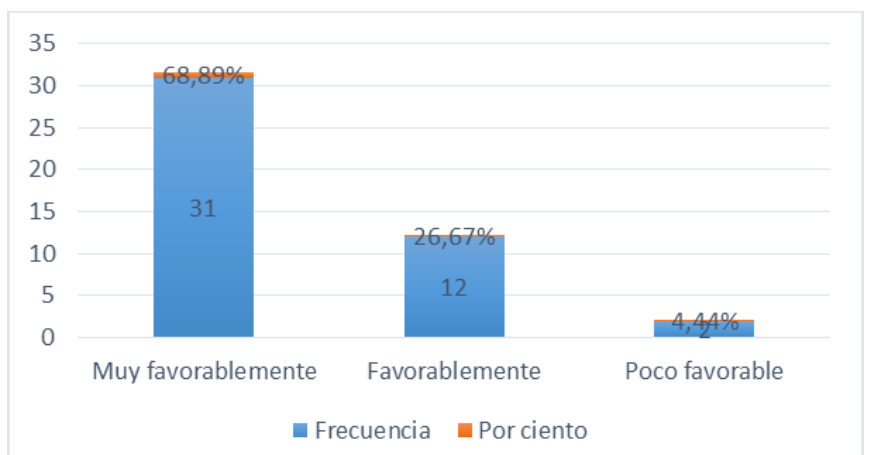

Figura 4. Incidencia de la profesionalización del sector educativo en los trabajadores de los CIBV

Al preguntar a los trabajadores de los CIBV cómo ha incidido en ellos el proceso de profesionalización del sector educativo llevado a cabo en todo el país, la mayoría ha contestado que el resultado ha sido muy favorable (31 para un 68,89\%), y mientras que para otros ha sido netamente favorable, 12 para un $26,67 \%$, para la gran minoría ha sido poco favorable ( 2 para un $4,44 \%)$.

Tabla 4. Incidencia de la profesionalización del sector educativo en los trabajadores de los CIBV

\begin{tabular}{lrr}
\hline Opciones & Frecuencia & Por ciento \\
\hline Muy favorablemente & 31 & $68,89 \%$ \\
Favorablemente & 12 & $26,67 \%$ \\
Poco favorable & 2 & $4,44 \%$ \\
\hline
\end{tabular}

Fuente: Elaboración propia

El Gobierno ecuatoriano ha invertido no solo recursos materiales al desarrollo integral de los infantes sino también recursos humanos a los cuales ha preparado para garantizar una atención de calidad a los infantes, pues el verdadero impacto de este tipo de programas recae en los seres humanos que trabajen en él, por tanto existe un reconocimiento por parte de los trabajadores de los CIBV de que su capacitación ha sido muy favorable, en tanto, la atención de 
los primeros años de vida de un niño no solo demanda afecto, sino atención a diversas necesidades y características específicas, planificar actividades, elaborar materiales didácticos y hacer buen uso de ellos, velar por la salud de los infantes, es decir, constituye un proceso complejo que requiere de competencias y habilidades que no afloran siempre de forma empírica sino que requiere de una instrucción profesional.

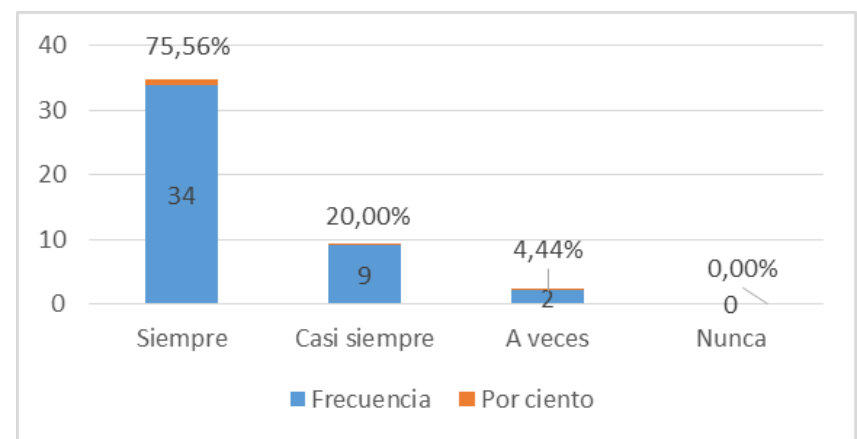

Figura 5. Buenas condiciones de infraestructura de los CIBV

La mayoría de los encuestados, 34 para un 75,56\%, está conforme con las condiciones de infraestructura de los CIBV, lo cual favorece a la primera infancia. Otro gran grupo de personas objetos de este estudio expresan que casi siempre tienen las condiciones de infraestructura garantizadas (9 para un 20\%), sin embargo, aunque no es una cifra estimable, para dos personas $(4,44 \%)$ la experiencia no es favorable en el aspecto de las condiciones de trabajo pues indican que solo a veces cuentan con la infraestructura en condiciones para realizar las actividades.

Tabla 5. Buenas condiciones de infraestructura de los CIBV

\begin{tabular}{lrr}
\hline Opciones & Frecuencia & Por ciento \\
\hline Siempre & 34 & $75,56 \%$ \\
Casi siempre & 9 & $20,00 \%$ \\
A veces & 2 & $4,44 \%$ \\
Nunca & 0 & $0,00 \%$ \\
\hline \multicolumn{2}{c}{ Fuente: Elaboración propia }
\end{tabular}

Lo que ha sucedido con las políticas públicas en el país relacionadas con el desarrollo infantil integral es que han contado con el financiamiento del Gobierno para proveer a los centros de las condiciones y parámetros necesarios para garantizar la atención a la primera infancia en un lugar acogedor, con el que la familia se sienta a gusto y a donde quiera regresar, lo cual constituye una puerta de éxito para el programa social de los CIBV.

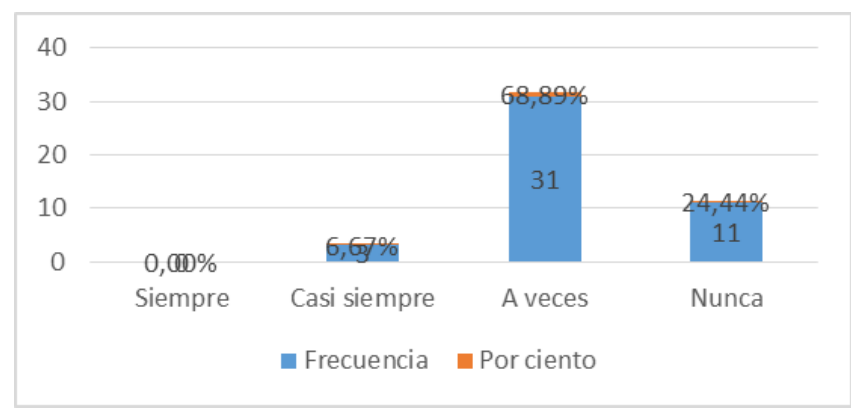

Figura 6. Cuenta con los medios didácticos los CIBV 
A pesar de contar con una favorable infraestructura, para la mayoría de los encuestados los Centros Infantiles del Buen Vivir se encuentran limitados pues solo cuentan con los medios didácticos a veces (31 para un 68,89\%), incluso algunos encuestados refieren no contar nunca con medios didácticos que no sean elaborados por ellos mismos ( 11 para un 24,44\%); solo en algunas instituciones de ese tipo se cuenta casi siempre con los medios didácticos que favorezcan la instrucción de los padres en el cuidado de los hijos (3 para un 6,67\%), ninguno de los consultados ha contado siempre con esos recursos.

Tabla 6. Cuenta con los medios didácticos los CIBV

\begin{tabular}{lrr}
\hline Opciones & Frecuencia & Por ciento \\
\hline Siempre & 0 & $0,00 \%$ \\
Casi siempre & 3 & $6,67 \%$ \\
A veces & 31 & $68,89 \%$ \\
Nunca & 11 & $24,44 \%$ \\
\hline
\end{tabular}

Fuente: Elaboración propia

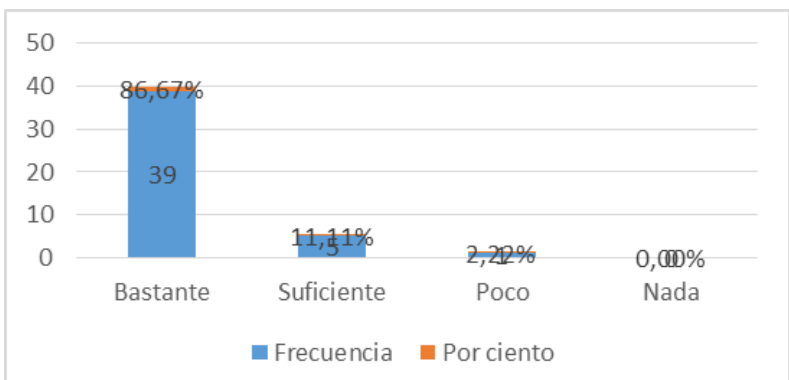

Figura 7. Influencia de los medios didácticos en la preparación de padres en CIBV

A pesar de saldo anterior, la gran mayoría de los trabajadores de los CIBV encuestados refieren que los medios didácticos tienen una gran influencia en su desempeño para lograr la capacitación de los padres y de los hijos (39 para un 86,67\%), para cinco personas $(11,11 \%)$ esos instrumentos de trabajo tienen una influencia suficiente, tal vez porque creen en que el mayor impulsor del cambio es el propio discurso del educador sin necesidad de valerse de alguna herramienta educativa. Solo un encuestado $(2,22 \%)$ refiere que es poca la influencia de los medios didácticos en la preparación de los CIBV, nadie reportó que sea nula la influencia de los medios educativos.

Tabla 7. Influencia de los medios didácticos en la preparación de padres en CIBV

\begin{tabular}{lrr}
\hline Opciones & Frecuencia & Por ciento \\
\hline Bastante & 39 & $86,67 \%$ \\
Suficiente & 5 & $11,11 \%$ \\
Poco & 1 & $2,22 \%$ \\
Nada & 0 & $0,00 \%$ \\
\hline \multicolumn{2}{c}{ Fuente: Elaboración propia }
\end{tabular}

Sin bien los educadores de los CIBV deben tener conocimientos y habilidades para la elaboración de medios didácticos que favorezcan el aprendizaje de los participantes en el programa de atención a la primera infancia, y de hecho la inventiva de los profesionales de la educación resuelven hoy múltiples carencias en determinadas actividades que conviene realizar, 
no es menos cierto que siempre resulta más atractivo y favorecedor al gusto de quienes reciben la capacitación contar con instrumentos que faciliten esas actividades, sobre todo porque no puede apelarse siempre al sentido de pertenencia de las educadoras y los padres sino que para el logro de ciertos objetivos los medios didácticos, como libros, productos audiovisuales y juguetes, resultan imprescindibles en la preparación y motivación de los propios actores impulsores del programa.

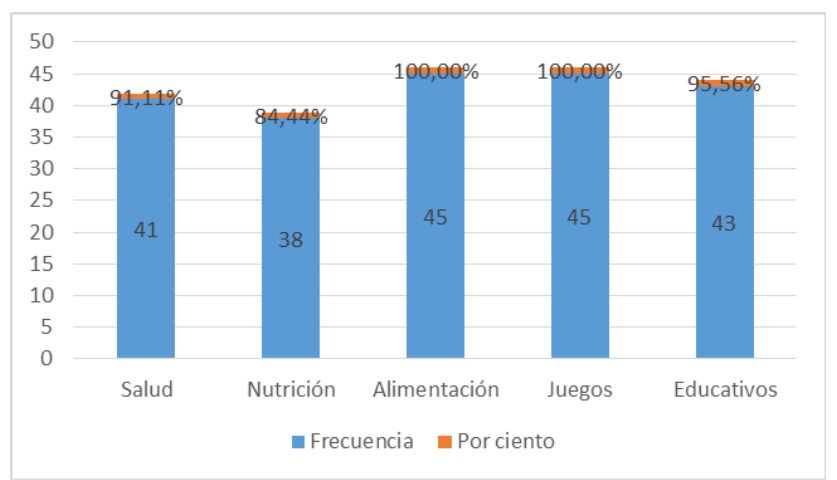

Figura 8. Temas que se tratan mejor con el empleo de los medios didácticos en los CIBV

Entre los temas que se tratan mejor con el empleo de los medios didácticos en los CIBV se encuentran el de alimentación (100\%) y los temas lúdicos (100\%), la educación (43 para un $95,56 \%$ ), salud (41 para un 91,11\%) y nutrición (38 para un 84,44\%) también resultan favorecidos con el empleo de herramientas educativas según la percepción de los encuestados.

Tabla 8. Temas que se tratan mejor con el empleo de los medios didácticos en los CIBV

\begin{tabular}{lrr}
\hline Opciones & Frecuencia & Por ciento \\
\hline Salud & 41 & $91,11 \%$ \\
Nutrición & 38 & $84,44 \%$ \\
Alimentación & 45 & $100,00 \%$ \\
Juegos & 45 & $100,00 \%$ \\
Educativos & 43 & $95,56 \%$ \\
\hline \multicolumn{2}{c}{ Fuente: Elaboración propia }
\end{tabular}

El tratamiento de asuntos como el de la salud, la alimentación, nutrición, educación y lo lúdico son temas priorizados en cada una de las políticas públicas que guardan relación con la primera infancia, pues el tratar estos asuntos ayuda a comprender mejor la necesidad de preservar los derechos de los infantes en tanto ayuda a prevenir problemáticas sociales como el hambre, la pobreza, la discriminación, el analfabetismo y el desempleo, que a su vez, como un ciclo, constituyen generadores de los problemas en la infancia. 


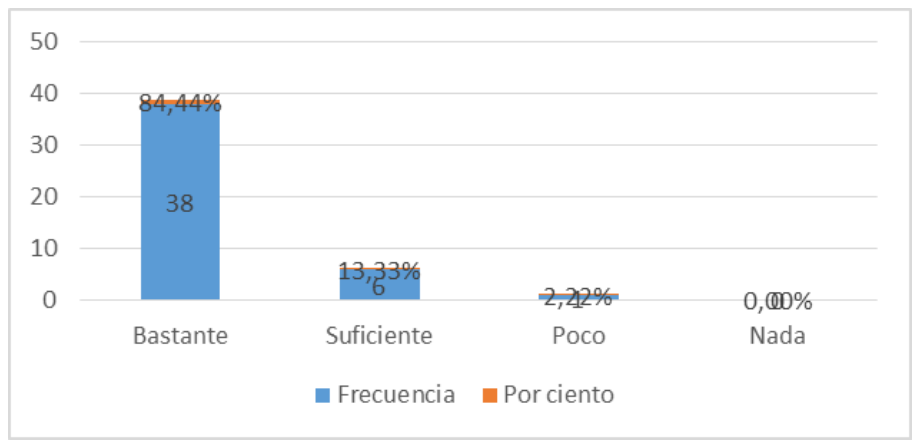

Figura 9: Nivel de prioridad a las familias con pobreza

Al preguntársele a los encuestados sobre el nivel de prioridad que les ofrecen a las familias de bajos recursos o índice de pobreza, los profesionales de los CIBV en su mayoría, 38 para un $84,44 \%$, respondieron ofrecerle bastante importancia, en seis casos se les ofrece suficiente prioridad y solo una persona opina que se le da poca prioridad.

Tabla 9. Nivel de prioridad a las familias con pobreza

\begin{tabular}{lrr}
\hline Opciones & Frecuencia & Por ciento \\
\hline Bastante & 38 & $84,44 \%$ \\
Suficiente & 6 & $13,33 \%$ \\
Poco & 1 & $2,22 \%$ \\
Nada & 0 & $0,00 \%$ \\
\hline
\end{tabular}

Fuente: Elaboración propia

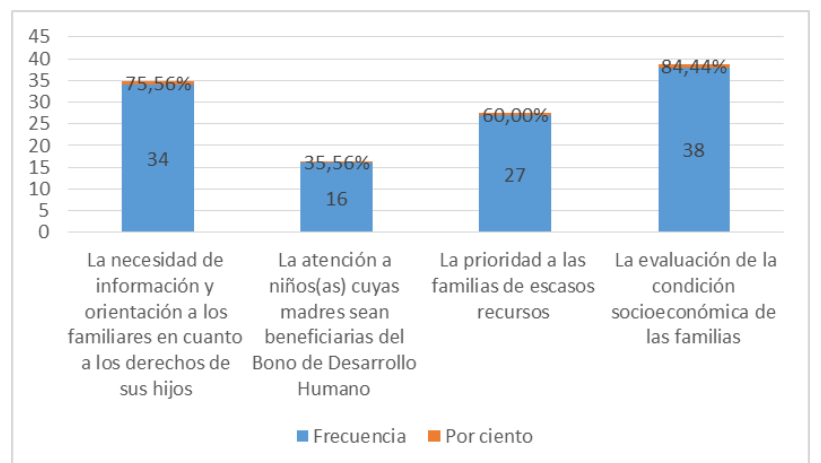

Figura 10: Motivos para la incorporación de las familias al programa de CIBV

Según el saldo de la encuesta la incorporación de la familia a los CIBV responde en primer lugar a la evaluación de la condición socioeconómica de los núcleos familiares (38 para un 84,44\%); en segundo lugar de la necesidad de información y orientación a los familiares en cuanto al derecho de sus hijos (34 para un 75,56\%), depende además de la prioridad a las familias con escasos recursos (27 para un 60\%) y de la atención que se le debe dar a los niños cuyas madres son beneficiarias del Bono de Desarrollo Humano (16 para un 35,56\%).

Tabla 9. Motivos para la incorporación de las familias al programa de CIBV

\begin{tabular}{lll}
\hline Opciones & $\begin{array}{l}\text { Frecuenc } \\
\text { ia }\end{array}$ & $\begin{array}{l}\text { Por } \\
\text { ciento }\end{array}$ \\
\hline
\end{tabular}




\begin{tabular}{lll}
\hline $\begin{array}{l}\text { La necesidad de información y orientación a los familiares en cuanto a los derechos } \\
\text { de sus hijos }\end{array}$ & 34 & $75,56 \%$ \\
La atención a niños(as) cuyas madres sean beneficiarias del Bono de Desarrollo & 16 & $35,56 \%$ \\
$\begin{array}{l}\text { Humano } \\
\text { La prioridad a las familias de escasos recursos }\end{array}$ & 27 & $60,00 \%$ \\
La evaluación de la condición socioeconómica de las familias & 38 & $84,44 \%$ \\
\hline
\end{tabular}

Fuente: Elaboración propia

Las políticas públicas cuyos beneficiarios son los niños han estado centradas en la intervención de las familias que sufren marcados problemas socioeconómicos como la marginalidad o discriminación, la pobreza, la desnutrición, enfermedades, violencia, de ahí que los planes de acción parten de un diagnóstico de las condiciones de vida de los participantes en estos programas, ello explica por qué para la captación de las familias se le ofrece bastante prioridad a los más pobres. Uno de estos ejemplos ha sido el proyecto de los CIBV.

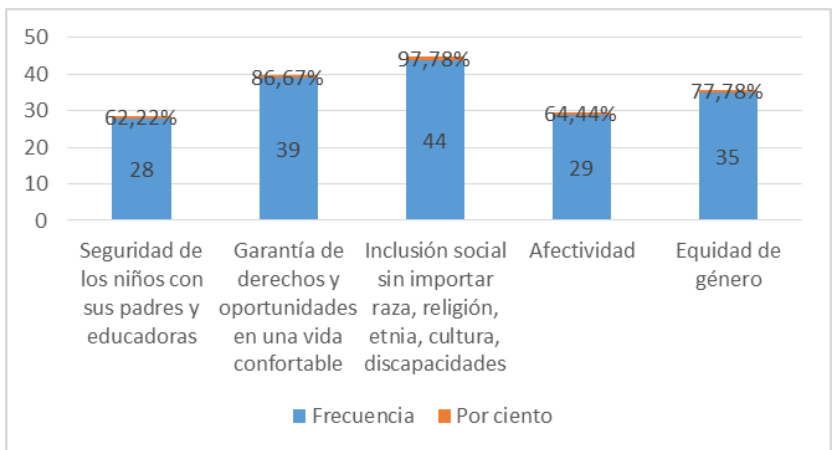

Figura 11: Niveles de prioridad a los objetivos de los CIBV

Según el resultado de esta Encuesta, aunque los educadores que trabajan en los CIBV laboran por alcanzar las metas de una sociedad más justa y garantizar el derecho a un desarrollo infantil integral, algunos ofrecen mayor prioridad a unos temas que a otros. De acuerdo con las perspectivas de los encuestados las actividades están más encaminadas con este orden de prioridad: garantizar la inclusión social sin importar raza, religión, etnia, cultura y discapacidades (44 para un 97,78\%); los derechos y oportunidades en una vida confortable (39 para un 86,67\%); ña equidad de género (35 para un 77,78\%); la afectividad (29 para un 64,44\%); y la seguridad de los niños con sus padres y educadores (28 para un 62,22\%).

Tabla11. Niveles de prioridad a los objetivos de los CIBV

\begin{tabular}{lcc}
\hline Opciones & Frecuencia & Por ciento \\
\hline Seguridad de los niños con sus padres y educadoras & 28 & $62,22 \%$ \\
Garantía de derechos y oportunidades en una vida confortable & 39 & $86,67 \%$ \\
Inclusión social sin importar raza, religión, etnia, cultura, discapacidades & 44 & $97,78 \%$ \\
& & $64,44 \%$ \\
Afectividad & 29 & $77,78 \%$ \\
Equidad de género & 35 &
\end{tabular}

El tema de la inclusión social constituye un importante asunto de la agenda gubernamental en un país como Ecuador, de una amplia diversidad cultural, religiosa, étnica. La firma de leyes, así como la implementación de las políticas públicas han tenido como eje central 
lograr la aceptación y el respeto a la diferencia. Contar con este tipo de pensamiento y conocimiento desde edades tempranas amplía las posibilidades de asegurar un futuro sin discriminación social en una sociedad que pretende ser más justa y desarrollada en todas las dimensiones de la vida, por lo que las personas consultadas para este estudio coinciden en que este es el objetivo de mayor prioridad y en el cual se concentran las actividades con los infantes.

\section{Discusión}

La publicación del Código de la Niñez y Adolescencia en 2003 en Ecuador, a pesar de que el país ya había trazado los primeros pasos en beneficio de la infancia, inauguró una nueva etapa con respecto a la perspectiva y las acciones que en relación a los derechos de los niños y niñas se implementarían.

Aunque el tema comenzó a formar parte de la agenda internacional desde la firma de la Convención de los Derechos del Niño, al que también accedió el Gobierno ecuatoriano desde 1990, no fue hasta bien entrado los años del nuevo milenio que el Estado ecuatoriano tomaría cartas en el asunto y comenzaría a promover políticas públicas que involucraría a las administraciones territoriales, comunidades, organizaciones de la sociedad civil e incluso al sector privado. La capacitación de la familia constituiría uno de los principales objetivos de esas políticas públicas dirigidas al desarrollo integral del infante, y aunque el presente estudio se concentra en el quehacer de los Centros Infantiles del Buen Vivir (CIBV), este objetivo se visualizaría también gracias a la incidencia del programa Creciendo con Nuestros Hijos $(\mathrm{CNH})$.

Este enfoque de integralidad por parte del Estado parte de la idea de entender que solo con una atención esmerada a todas dimensiones de la vida de los niños y niñas existen más posibilidades de lograr en ellos un desarrollo integral, definido por la UNICEF (2016) como el "desarrollo físico, cognitivo, lingüístico y socio-emocional de los niños y niñas de 0 a ocho años, de una manera integral para el cumplimiento de todos sus derechos" (p. 1); aunque en Ecuador la primera infancia se concibe hasta los cinco años.

Esta nueva interpretación de lo que es desarrollo integral de la infancia ha sido bien acogida por los especialistas que laboran en los CIBV que tienen a su cargo la orientación a las familias sobre la atención a los hijos. Las personas encuestadas reconocen que no solo con la atención específica a áreas como la salud, nutrición, alimentación o la educación se consigue el desarrollo pleno de los pequeños, ello solo se logra con una implementación con enfoque holístico de las actividades, pues en el crecimiento de los infantes no solo influyen los aspectos físicos sino también el contexto en el que sucede la crianza.

Al referirse al tema Moreno (2016) ha señalado que solo una infancia bien protegida garantizará una semejante vida adulta, de manera que si el país pretende contar con una sociedad más desarrollada, conformada por individuos con mejores habilidades y competencias para el trabajo, con una perspectiva de vida más duradera y con una mejor percepción sobre su medio ambiente, debe emerger una atención exhaustiva a la infancia de esas personas.

En ese sentido, ha tomado "un lugar preponderante la recuperación de la familia como promotora de la integración social (UNICEF, 2012, p. 11), y las personas consultadas coinciden 
en que la preparación de los profesionales de los CIBV a los padres y cuidadores de los niños ha incidido muy favorablemente en la calidad de vida y desarrollo integral de los infantes, pues es la familia la institución que más incide en la vida de los pequeños (Berlinski \& Schady, 2015), son los progenitores quienes deciden cómo se alimenta y nutre el hijo, cuándo es necesario visitar al médico, qué aspectos debe aprender el pequeño; por tanto, influir en las prácticas de crianza de los adultos es incidir en un crecimiento sano y desarrollado de los niños y niñas.

Quizás por ello es que un gran por ciento de los encuestados opinó que el desarrollo integral depende de los padres, y es que la trasferencia de afecto en los primeros años de vida, según reportan los estudios, resulta determinantes para el desarrollo del cerebro y la transmisión de valores a medida que el pequeño crece. De ahí que muchos de los programas de atención a la infancia que han tenido éxito en la región han entendido a la familia como su principal aliada para visibilizar el cambio de las prácticas de la crianza centrados sobre todo en la estimulación cognitiva precoz. No obstante, sobre la familia también recaen problemas socioeconómicos como el divorcio, la pobreza, el hambre, el desempleo, la discriminación, la marginalidad, que atenta contra la crianza de los hijos.

Por ello, las políticas públicas en relación a la niñez primera, si bien se centran en el trabajo con la familia, defienden el enfoque intersectorial de las estrategias y acciones implementadas, como garantía para el éxito, como es también la opinión de la mayoría de los encuestados. "Ello supone el desafío de establecer los acuerdos y consensos necesarios para que la articulación intra e intersectorial nacional y la gestión sean una realidad" (UNICEF, 2012, p. 11), pues implica desde contar con trabajo para los padres hasta garantizar la alimentación de las comunidades, la calidad de los servicios de los centros de salud, la accesibilidad a la escuela, la seguridad ciudadana.

El Estado, con su papel regulador de las prácticas de la sociedad, advierte que el mejor camino es el de "fortalecer el rol de la familia y la comunidad en la crianza de sus hijos e hijas" (Espinel, 2016, p. 1). Ello sería hoy imposible sin la incidencia, por un lado, de los espacios de cuidado de la primera infancia en los Centros Infantiles del Buen Vivir; así como la existencia técnica, capacitación y asistencia financiera a esas instituciones enclavadas en la comunidad.

De acuerdo a los resultados de la investigación, estas instalaciones cuentan con la infraestructura necesaria para ofrecer un ambiente confortable de los padres y niños que participan en el programa, entendiendo ello, como lo que expresara Moreno (2016) en su estudio:

La existencia de áreas de recreación al aire libre; la existencia de, al menos, un 30\% de espacio dedicado únicamente para la movilidad de los infantes, los juegos y las actividades; la existencia de lavamanos e inodoros con altura especial para niños; la existencia de un área exclusiva de alimentación, de un área exclusiva para el control de la salud y de un área exclusiva de cocina; el correcto funcionamiento de los servicios básicos y la división de los espacios de atención de acuerdo a los grupos de edad de los infantes (p. 71).

A pesar de los recursos disponibles para el desarrollo de este programa, según los encuestados no satisfacen los medios didácticos para la auto preparación de las educadoras de esos centros y ejercer la capacitación de la familia en el cuidado de sus hijos. No obstante, existe 
la percepción de la importancia de las herramientas educativas para tratar la amplia variedad temática que suponen esos centros, pues al decir de la UNICEF (2012) conviene que la familia conozca sobre:

Lactancia materna, alimentación y nutrición, cuidados en el embarazo y del infante, prevención de enfermedades infantiles, hábitos de higiene, educación sexual integral, lectura y narración, juego, aprendizajes tempranos, escolarización inicial, promoción del trabajo decente, erradicación del trabajo infantil, derechos del niño, integración de niños con necesidades especiales, perspectiva de género, violencia familiar de género, ciudadanía y participación comunitaria, derecho a la identidad y diversidad cultural y comunicación comunitaria, entre otros (p. 12).

Sin embargo, el tratamiento de estas temáticas se ve limitadas por no contar con los medios didácticos que favorezcan un mejor entendimiento respecto a esos asuntos, entre ellos se encuentran los libros y las revistas que tratan asuntos sobre la atención infantil, productos audiovisuales que muestran metodologías y contextos reales de las actividades que se pueden realizar con los niños; así como los juguetes que son empleados para desarrollar habilidades y capacidades en los pequeños.

A pesar de estas limitaciones, uno de los logros que se alcanza con la implementación de las políticas públicas ha sido el carácter inclusivo de las estrategias y las actividades desarrolladas en función del desarrollo integral del niño en Ecuador. En primer lugar, por la prioridad que se ofrece a las familias con bajo índice económico o que presentan problemáticas sociales en su seno.

La creación de los Centros Infantiles del Buen Vivir va a tono con esta idea de promover la inclusión de los niños, teniendo en cuenta la diversidad cultural de una sociedad como la ecuatoriana. De acuerdo con los resultados de la Encuesta implementada, la captación de las familias beneficiadas por esas instalaciones parte de la evaluación de la condición socioeconómica de los núcleos familiares, y en este sentido, se les ofrece a las familias de bajos recursos o niveles de pobreza mayor prioridad.

A este buen saldo, se le suma las observaciones realizadas por Moreno (2016) en su investigación empírica al comprobar la existencia en los CIBV de niños blancos, mulatos, mestizos, entre otros. En cuanto a la asistencia de niños discapacitados "solo en el $40 \%$ se pudo comprobar la asistencia de niños con necesidades educativas especiales, y en todos ellos, las educadoras afirmaron haber recibido instrucción previa de cómo fortalecer la atención hacia estos infantes" (p. 75).

Contar con instituciones como los Centros Infantiles del Buen Vivir, desde el momento en punto en que incluye y promueve la participación de todos los niños independientemente de sus diferencias culturales y necesidades educativas especiales, constituye una experiencia positiva que agiganta pasos en relación al aprendizaje de los niños a través de actividades pedagógicas y lúdicas que desarrollan valores morales, el respeto a la diversidad, la equidad de género. 
Las políticas públicas que inciden en la actualidad en el desarrollo integral de la primera infancia en Ecuador tienen un importante actor, que es el educador o cuidador de los centros especializados en la atención a los niños y niñas, por tanto, la inversión deberá estar dirigida en mantener la capacitación continua a estos profesionales, en un afán por ayudar a la familia a la crianza de sus hijos, respetando el derecho a la oportunidad de una vida saludable y confortable, como garantía de las generaciones futuras que conforman una sociedad en desarrollo.

\section{Conclusiones}

Si bien en la última década del siglo XX Ecuador comenzó a hacerse eco de las acciones internacionales en función del respeto a los derechos de la infancia, no fue hasta entrada la primera década del nuevo milenio que el país comenzaría a priorizar las leyes y políticas públicas que abogaran por el desarrollo integral de los niños y niñas, hasta convertirse en la actualidad en uno de los países que más se destacan en este frente social.

El estudio de la incidencia de los Centros Infantiles del Buen Vivir en el cuidado de la vida de los niños permite corroborar cómo solo con el involucramiento de varios sectores de la comunidad como las instituciones de salud y educación, empresas, organizaciones de la sociedad civil, se puede lograr un desarrollo pleno en los primeros años de vida. No obstante, para ello resulta imprescindible contar con los recursos y medios didácticos que favorezcan la capacitación continua de aquellas educadoras y cuidadores que se encargan, de forma paralela a la familia, del cuidado de los niños.

Uno de los principales objetivos de las políticas públicas llevadas a cabo en Ecuador ha sido fomentar la preservación de los derechos de la niñez, y los planes de acción que se implementen en este sentido debe tener presente principios como la equidad de género y la inclusión, además de mantener una prioridad a aquellas niñas y niños que pertenecen a familias en condiciones de pobreza o que sufren de otros problemas socioeconómicos como el desempleo, la marginalidad, el analfabetismo, la violencia, la discriminación. Sin embargo, debe predominar el trabajo intersectorial para el verdadero éxito de programas sociales de este tipo.

\section{Bibliografía}

Berlinski, S., \& Schady, N. (2015). Los primeros años. El bienestar infantil y el papel de las políticas públicas. New York: Banco Interamericano de Desarrollo.

Cáceres, C. (2011). Hitos Históricos del Desarrollo Infanitl en el Ecuador. Recuperado de La Chakana: http://psicologiaporlavida.blogspot.com/2011/02/hitos-historicos deldesarrollo.html

Carneiro, P., \& Heckman, J. J. (2003). Human Capital Policy. En J. J. Heckman, \& A. B.

Krueger, Inequality in America: What Role for Human Capital Policies? Cambridge: MA: MIT Pres. 
Comité de los Derechos del Niño. (2006). Realización de los derechos del niño en la primera infancia. Observación General. Ginebra: Convención sobre los Derechos del Niño.

Dodge, D. (2015). Capital Humano, Desarrollo de la Primera Infancia y Crecimiento Económico.

Enciclopedia sobre el Desarrollo de la Primera Infancia, 7-8.

Espinel, M. (2 de julio de 2016). Primera Infancia. Recuperado de Ministerio Coordinador del Desarrollo Social: http://www.desarrollosocial.gob.ec/estrategia-nacionalintersectorial para-la-primera-infancia-infancia-plena

Lowe, R. (2004). Childhood through the Ages. En T. Maynard, \& N. Thoma, An Introduction to Early Childhood Studies. Londres: SAGE Publications Ltd.

MIES. (2013). Política Pública Desarrollo Infantil Integral. Quito: Ministerio de Inclusión Económica y Social.

Moreno, C. (2016). Análisis de la política de desarrollo infantil integral, en niñas y niños de 0 a 3 años de edad, en centros infantiles del buen vivir (CIBV). Quito: Universidad Andina Simón Bolívar.

ODNA. (2012). Estado de los Derechos de la Niñez y la Adolescencia en Ecuador 1990-2011.

Quito: Observatorio de los Derechos de la Niñez y Adolescencia.

Olavarría, M. (2007). Conceptos Básicos en el Análisis de Políticas Públicas. Instituto de Asuntos Públicos de la Universidad de Chile: Instituto de Asuntos Públicos de la Universidad de Chile.

SENPLADES. (2015). Ecuador enfoca su atención en la primera infancia. Recuperado de Secretaria Nacional de Planificación y Desarrollo: http://www.planificacion.gob.ec/ecuador-enfoca-su-atencion-en-la-primera-infancia/

UNESCO. (2012). Derechos desde el principio. París: UNESCO.

UNICEF. (2010). Para la Vida. Nueva York: UNICEF. Recuperado de http://www.unicef.org/spanish/earlychildhood/index_3870.html

UNICEF. (2012). Crecer juntos para la primera infancia. encuentro regional de políticas integrales 2011. Buenos Aires: UNICEF.

UNICEF. (2016). Convención sobre los Derechos del Niño. Obtenido de UNICEF: http://www.unicef.es/infancia/derechos-del-nino/convencion-derechos-nino 


\section{Anexos}

1. ¿Los niños y niñas que reciben una adecuada atención durante su primera infancia, aumentan sus oportunidades de desarrollo integral?
Siempre
Casi siempre

A veces

Nunca

2. De acuerdo a las evaluaciones ¿Cómo ha incidido la preparación de los profesionales de los CIBV a los padres y cuidadores de los niños en la calidad de vida y desarrollo integral de los infantes?

Muy favorable Favorablemente

Poco favorable

3. El desarrollo integral de los niños depende de:

Los padres

Los docentes
Los médicos Un trabajo intersectorial

4. El país ha iniciado un proceso de profesionalización del sector educativo, ¿cómo ha influido ello en los especialistas de CIBV?

Muy favorable

Favorablemente

Poco favorable

5. Cuenta el CIBV con las condiciones de infraestructura para favorecer a la primera infancia Siempre Casi siempre A veces Nunca

6. ¿Cuenta el CIBV con los medios didácticos elementales para promover la educación de padres de niños que están en la primera infancia?
Siempre
Casi siempre
A veces
Nunca

7. ¿Cuánto influyen los medios didácticos en la preparación de padres de niños que están en la primera infancia?

Bastante

Suficiente

Poco

Nada

8. ¿Qué temas se tratan mejor con el empleo de los medios didácticos en los CIBV?

Salud

Nutrición

Alimentación

Juegos

Educativo

9. a) Qué nivel de prioridad les ofrecen a las familias de bajos recursos o niveles de pobreza Bastante Suficiente Poco Nada

b) De qué depende la incorporación de la familia a los CIBV hijos

La necesidad de información y orientación a los familiares en cuanto a los derechos de sus

_ La atención a niños(as) cuyas madres sean beneficiarias del Bono de Desarrollo Humano

La prioridad a las familias de escasos recursos

La evaluación de la condición socioeconómica de las familias

10. Qué nivel de prioridad les ofrece a los objetivos de los CIBV

Seguridad de los niños con sus padres y educadoras

Garantía de derechos y oportunidades en una vida confortable

Inclusión social sin importar raza, religión, etnia, cultura, discapacidades

Afectividad

Equidad de género 